\title{
A New Spaceborne Burst Synthetic Aperture Radar Imaging Mode for Wide Swath Coverage
}

\section{Pingping Huang ${ }^{1, *}$ and Wei $\mathrm{Xu}{ }^{2}$}

1 College of Information Engineering, Inner Mongolia University of Technology, Hohhot 010051, China

2 Department of Spaceborne Microwave Remote Sensing, Institute of Electronics, Chinese Academy of Sciences (IECAS), Beijing 100190, China; E-Mail: iecasxuwei@gmail.com

* Author to whom correspondence should be addressed; E-Mail: hwangpp@imut.edu.cn; Tel.: +86-186-8606-2012.

Received: 12 December 2013; in revised form: 2 January 2014 / Accepted: 3 January 2014 / Published: 13 January 2014

\begin{abstract}
This paper presents a new spaceborne synthetic aperture radar (SAR) burst mode named "Extended Terrain Observation by Progressive Scans (ETOPS)" for wide swath imaged coverage. This scheme extends the imaging performance of the conventional Terrain Observation by Progressive Scans (TOPS) mode with a very limited azimuth beam steering capability. Compared with the TOPS mode with the same azimuth beam steering range for the same swath width, a finer azimuth resolution could be obtained. With the same system parameters, examples of four burst SAR imaging modes named ScanSAR, TOPS, inverse TOPS (ITOPS) and ETOPS are given, and their corresponding system performances are analyzed and compared. Simulation results show that the proposed ETOPS mode could obtain a better high-resolution wide-swath imaging performance under the same conditions.
\end{abstract}

Keywords: synthetic aperture radar (SAR); Terrain Observation by Progress Scans (TOPS); wide swath coverage; performance analysis

\section{Introduction}

Wide unambiguous swath coverage is one of most important aims for future spaceborne synthetic aperture radar (SAR) missions [1]. The burst mode is a sophisticated imaging scheme which can achieve very wide swath coverage by periodically switching the antenna elevation beam to multiple 
sub-swaths [1-4]. ScanSAR is a conventional burst mode for wide swath coverage, which reduces the burst duration to less than the synthetic aperture time in order to illuminate other range sub-swaths. Wide swath coverage makes ScanSAR quite attractive, particularly for its wide swath interferometry application. ScanSAR interferometry made it possible to map $80 \%$ of the earth's landmass during the 11-day Shuttle Radar Topography Mission [4,5]. In addition to the impaired azimuth resolution, targets are illuminated by different parts of the azimuth antenna pattern (AAP) depending on their azimuth locations in the ScanSAR burst. This scheme leads to azimuth variant ambiguity to signal ratio (ASR) and signal to noise ratio (SNR), known as scalloping effect, all of which impair the SAR image quality or affect the follow on image processing, especially for the subsequent interferometric evaluation [6].

The Terrain Observation by Progressive Scans (TOPS) mode is a novel burst imaging mode for wide swath coverage [6-9]. It achieves wide swath coverage by periodically switching the antenna beam from sub-swath to sub-swath, similar to conventional ScanSAR. In azimuth, the antenna beam is actively steered from aft to fore with the opposite direction to the spotlight case during the burst duration interval. Consequently, targets with different azimuth locations are illuminated by the completed azimuth antenna pattern (AAP). The primary drawbacks in conventional ScanSAR images are obviously reduced and overcome [6,9]. The TOPS mode was first demonstrated by the TerraSAR-X satellite and has been chosen as the default mode for the European Space Agency (ESA)'s Sentinel-1 system for interferometric wide swath imaging [9-11].

In TOPS, the azimuth beam is usually electronically scanned from aft to fore during the whole acquisition interval. However, the finite azimuth beam steering capacity in the recent spaceborne SAR missions, for example, $\pm 0.75^{\circ}$ in the TerraSAR-X and $\pm 0.7^{\circ}$ in the Sentinel-1case [9-11], reduces the obtained azimuth resolution. To improve the azimuth resolution in the case of the limited azimuth steering capacity, a novel extended TOPS (ETOPS) mode is proposed in this paper. Based on the same azimuth beam scanning capacity, an improved azimuth resolution can be designed in the proposed ETOPS mode, since the azimuth beam pointing direction is fixed during part of the burst acquisition time. With the same system parameters, four imaging modes (ScanSAR, TOPS, inverse TOPS and ETOPS) are designed, and their corresponding system performances are analyzed and compared.

This paper is arranged as follows. Section 2 briefly reviews the TOPS mode, while the residual scalloping effect and other slightly azimuth variant system performances are analyzed and compared with conventional ScanSAR. A novel spaceborne ETOPS mode is proposed in Section 3. With the same system parameters, four different burst imaging modes are designed and compared in Section 4. Finally, some conclusions are reported in Section 5.

\section{TOPS Imaging Mode}

\subsection{TOPS Mode Review}

The TOPS mode requires the sensor antenna beam to be steered in both elevation and azimuth. In elevation, the antenna beam scans cyclically from sub-swath to sub-swath, using the burst mode similar to ScanSAR; in azimuth, the azimuth antenna beam is swept from aft to fore to shrink the target illumination time, as shown in Figure 1. In Figure 1, $t$ defines the slow time axis (in azimuth), $\omega_{r}$ is the 
azimuth beam rotation rate, $v_{s}$ is the velocity of the satellite. By taking the azimuth beam rotation into account, echoes of each target are weighted by a reduced AAP. The new AAP can be regarded as [2]:

$$
G_{T O P S}(t) \approx G_{0} \cdot \operatorname{sinc}^{2}\left[\frac{L_{a}}{\lambda} \frac{v_{g} t}{r}\left(1+\frac{\omega_{r} r}{v_{g}}\right)\right]
$$

where $G_{0}$ is a constant representing the gain of the AAP, $L_{a}$ represents the length of the azimuth antenna, $v_{g}$ is the velocity of the footprint without taking azimuth beam steering into account, $r$ is the slant range. $G_{T O P S}(t)$ can be equivalent to the AAP of a fixed antenna in the classical stripmap mode, but shrunk by a factor $A$ :

$$
A(r)=1+\omega_{r} r / v_{g}
$$

This factor shrinks the target illumination time and is responsible for the coarser azimuth resolution [2]. As each target experiences the completed AAP weighting in TOPS, azimuth varying system performances in conventional ScanSAR are overcome.

Figure 1. Terrain Observation by Progressive Scans (TOPS) mode acquisition geometry.

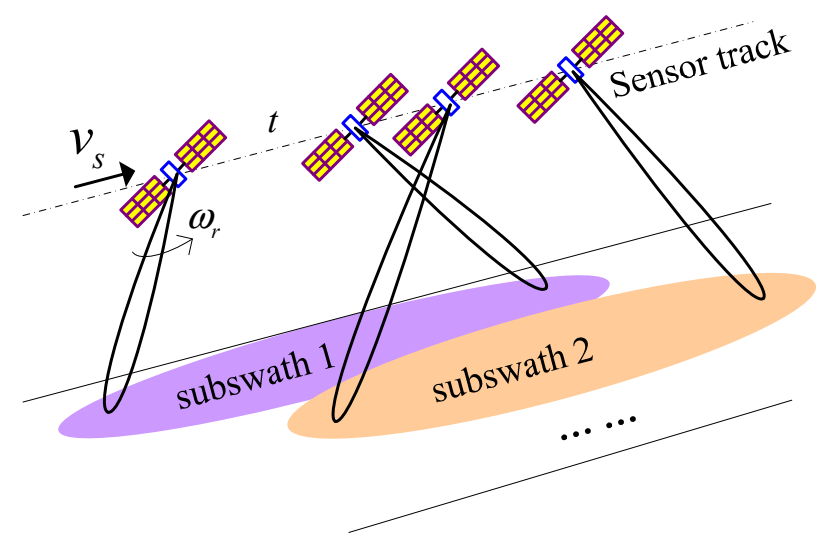

Since the AAP is symmetrical, the shrunk AAP can also be obtained by steering the azimuth beam from fore to aft, the same direction as the spotlight mode, but with a higher beam rotation rate. This scheme is named as the inverse TOPS (ITOPS) mode. With the same antenna, in order to obtain the same azimuth resolution, the azimuth beam rotation rate $\omega_{i r}$ in the ITOPS mode is expressed as follows:

$$
\omega_{i r}=-\left(\frac{2 v_{g}}{r}+\omega_{r}\right)
$$

\subsection{Performance Analysis in Azimuth}

To implement progressive beam scanning in both azimuth and elevation, a two-dimensional (2D) planar phased array antenna is usually adopted for the spaceborne SAR missions. The two-way azimuth antenna pattern of the phased array antenna can be written as follows: $[12,13]$ :

$$
G(\theta, t)=G_{e}(\theta) \cdot\left|\frac{1}{N} \sum_{k=0}^{N-1} C_{k, T}(t) \exp \left(j \frac{2 \pi k}{\lambda} L_{a e} \sin \theta\right)\right| \cdot\left|\frac{1}{N} \sum_{k=0}^{N-1} C_{k, R}(t-\Delta t) \exp \left(j \frac{2 \pi k}{\lambda} L_{a e} \sin \theta\right)\right|
$$


with

$$
\begin{gathered}
G_{e}(\theta)=G_{0} \cdot \operatorname{sinc}^{2}\left(\frac{L_{a e}}{\lambda} \sin \theta\right) \\
C_{k, T}(t)=a_{k, T} \cdot \exp \left[j \frac{2 \pi k}{\lambda} L_{a e} \sin \left(\theta_{p}(t)\right)\right] \\
C_{k, R}(t)=a_{k, R} \cdot \exp \left[j \frac{2 \pi k}{\lambda} L_{a e} \sin \left(\theta_{p}(t-\Delta t)\right)\right]
\end{gathered}
$$

where $G_{e}(\theta)$ is the antenna pattern of the antenna element, $N$ is the number of Transmit/Receive (T/R) modules in azimuth, $L_{a e}$ is the length of one antenna element, $C_{k, T}(t)$ and $C_{k, R}(t)$ are the excitation coefficients of the transmit and receive azimuth beam steering law, respectively, $a_{k, T}$ and $a_{k, R}$ are transmit and receive excitation coefficients amplitudes, respectively, $\Delta t$ is the delay time for the radar echo reception, and $\theta_{p}(t)=\omega_{r} t$ is the steering angle, which varies with the slow time $t$. Figure 2 shows the array antenna pattern, and it indicates that the grating lobe energy is increased and the main lobe gain is reduced during the antenna beam steering, when the element antenna length is longer than the wavelength. In this simulation, the frequency carrier is $9.65 \mathrm{GHz}$, and the antenna length is $6.4 \mathrm{~m}$. With the increased steering angle, the grating lobes arise and the main lobe gain reduces. This shows that the azimuth beam steering capacity is limited for spaceborne SAR systems, and it is the reason for a small scalloping effect, as well as the slight azimuth variation of signal to noise (SNR) and ambiguity to signal ratio (ASR) in TOPS [14].

Figure 2. Antenna beam steering. (a) Grating lobes; (b) Main lobe reduction.

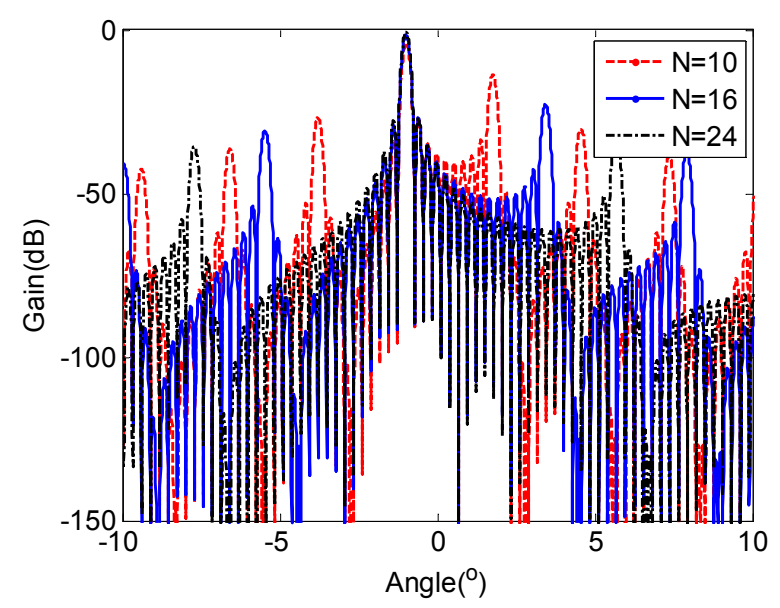

(a)

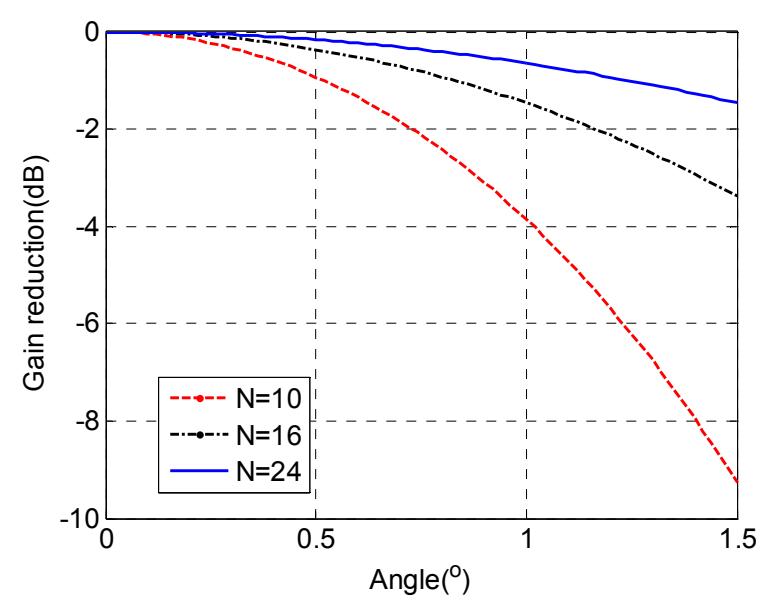

(b)

As azimuth burst working principles of ScanSAR and TOPS are quite different as shown in Figure 3, the two modes share different system performances. In ScanSAR, the whole synthetic aperture time of the stripmap case is divided into multiple segments to illuminate different sub-swaths; the whole synthetic aperture time of the stripmap case is compressed via steering the azimuth beam from aft to fore in TOPS. Echoes of targets at different azimuth locations are weighted by different parts of the AAP in ScanSAR, while echoes of all targets in TOPS share the complete AAP and the small difference is caused by the azimuth element antenna pattern (AEAP) as shown in Figure $3 b$. 
Figure 3. Different azimuth burst imaging schemes in ScanSAR and TOPS. (a) ScanSAR; (b) TOPS SAR. (SAR = synthetic aperture radar)

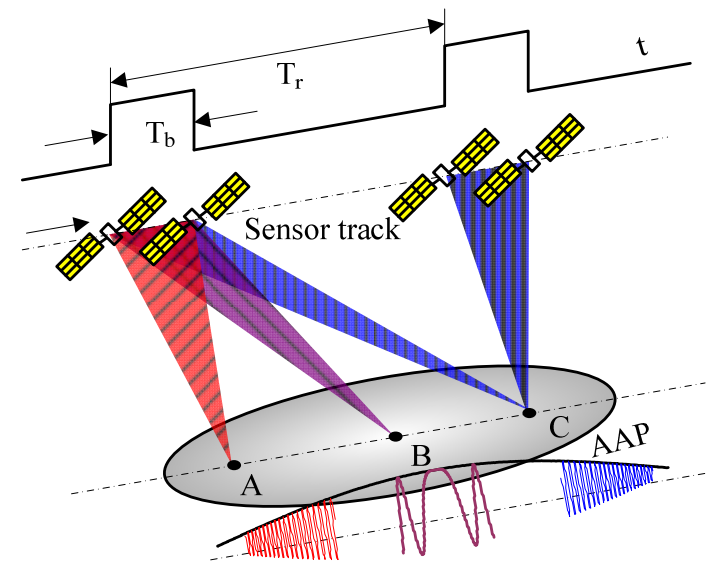

(a) ScanSAR

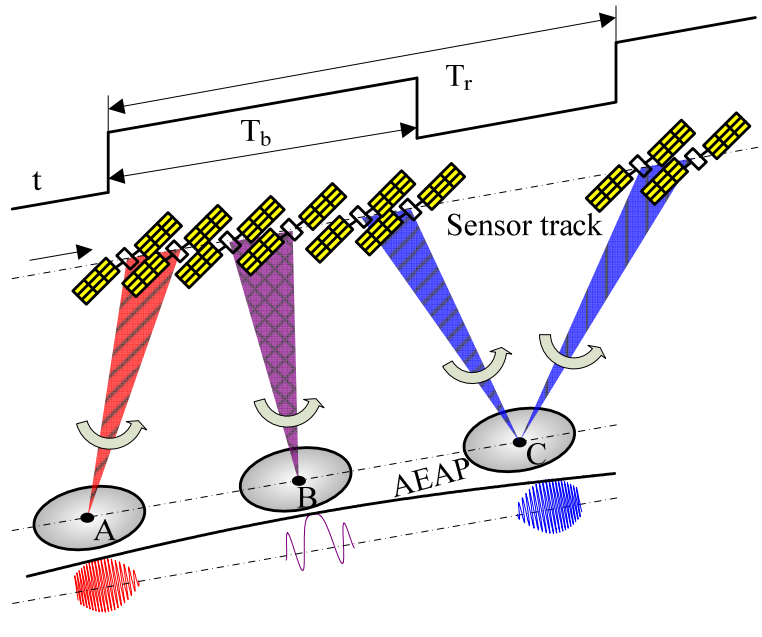

(b) TOPS SAR

The $k$-th grating lobe is located at angles:

$$
\varphi-\varphi_{0}= \pm k \lambda / L_{a e}
$$

where $\varphi_{0}$ is the azimuth squint angle. As signal and ambiguity energy are integrated in the focusing angular interval, the system AASR can be computed as follows [12]:

$$
\mathrm{AASR}=\frac{\sum_{m \neq 0} \int_{-\vartheta_{0} / 2}^{\vartheta_{0} / 2} G^{2}\left(\varphi-m \cdot \vartheta_{a m b}\right) d \varphi}{\int_{-\vartheta_{0} / 2}^{\vartheta_{0} / 2} G^{2}(\varphi) d \varphi}
$$

where $\vartheta_{0}$ is the focusing angular interval, $\vartheta_{a m b}$ is the interval angle between two ambiguity directions and is described as:

$$
\vartheta_{a m b}=\frac{\lambda \cdot \mathrm{PRF}}{2 v_{s}}
$$

The main azimuth ambiguity energy will be introduced by the grating lobe and the AASR will become worse, if the system PRF is selected as follows:

$$
\mathrm{PRF}=\frac{2 k v}{m L_{a e}}, \quad k, m \in\{1,2,3, \ldots, N\}
$$

Since the first pair of grating lobes has the highest energy power, the case of $k=1$ should be considered during the TOPS mode system design. Figure 4 shows simulation results of AASR varying with the PRF, the squint angle of Figure $4 \mathrm{~b}$ is $0.7^{\circ}$ and the azimuth antenna is $6.4 \mathrm{~m}$ consisting of 16 elements. For the increased squint angle grating lobes arise, and Figure 4 illustrates their impact on the AASR.

In addition to the impact of grating lobes on the AASR, the effect of main lobe gain reduction on the SNR should be considered during the system design. The effect can be expressed by the azimuth loss $L_{a z}$ given as: 


$$
L_{a z}=\frac{\int_{-B_{d} / 2+f_{D C}}^{B_{d} / 2+f_{D C}}\left|H_{p}\left(f_{a}\right)\right|^{2} d f_{a}}{\int_{-B_{d} / 2+f_{D C}}^{B_{d} / 2+f_{D C}}\left|G_{a}\left(f_{a}\right)\right|^{2} \cdot\left|H_{p}\left(f_{a}\right)\right|^{2} d f_{a}}
$$

where $H_{p}\left(f_{a}\right)$ is the transfer function of the azimuth processing filter, $B_{d}$ is the target Doppler bandwidth, $f_{D C}$ is the target Doppler centroid, $G_{a}\left(f_{a}\right)$ is the joint Tx-Rx azimuth antenna pattern function which is weighting the Doppler spectrum.

Figure 4. Azimuth ambiguity to signal ratio (AASR) varying with the pulse repetition frequency (PRF). (a) With different steering angles; (b) With a steering angle of $0.7^{\circ}$.

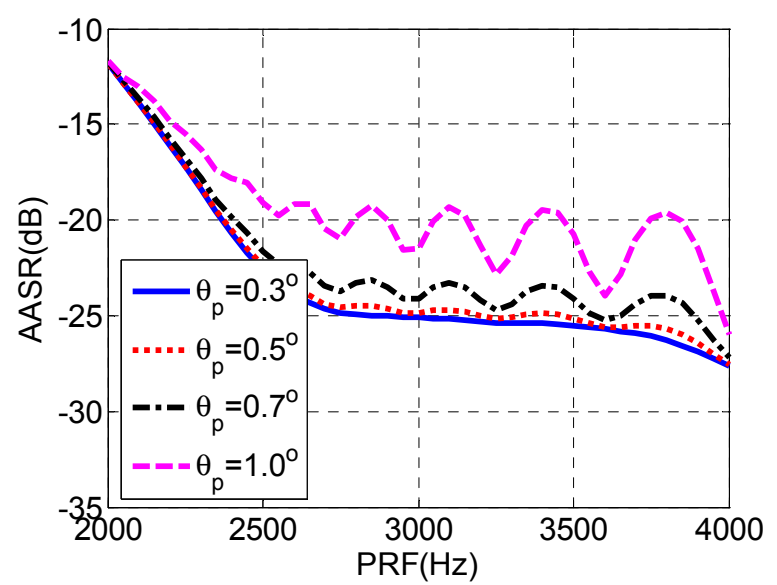

(a)

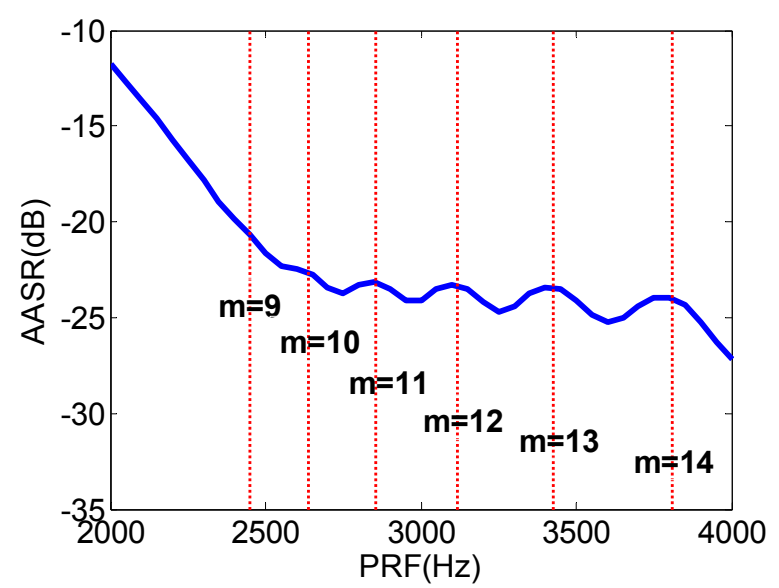

(b)

From Equations (9) and (12), it can be seen that the AAP will affect the system performances AASR and the azimuth loss, and Figure 5 demonstrates this effect. If more antenna elements are adopted for a fixed antenna length, the effect on grating lobes and main lobe reduction will be reduced. This results in a better system performance.

\subsection{Timeline Design in TOPS}

For burst imaging schemes, different sub-swaths are periodically illuminated in elevation, and the timeline design becomes very important to keep the imaged area continuous in both azimuth and elevation. After computing the azimuth beam steering rate in the $i$-th sub-swath, the TOPS timeline could be derived. The constraint of the TOPS timeline results in a set of $n$ linear equations as follows $[6,9]$ :

$$
\left(\omega_{r_{-} i} T_{b_{-} i}-\vartheta_{0}\right) R_{i}+v_{g} T_{b_{-} i}=(1+\varepsilon) v_{g} \sum_{i}^{N} T_{b_{-} i}=(1+\varepsilon) v_{g} T_{r}
$$

where $T_{b_{-} i}$ is the burst duration of the $i$-th sub-swath, $\vartheta_{0}$ is the azimuth beam interval exploited for focusing, $R_{i}$ is the closest slant range, $\varepsilon$ is the overlap ratio between two adjacent imaged areas in azimuth, and $T_{r}$ is the cycle time. Figure 6 shows the proper TOPS timeline in the azimuth time-frequency diagram for keeping the imaged area continuous in azimuth, while $\mathrm{A}$ and $\mathrm{C}$ are targets at the edge of the imaged area of a burst. In Figure 6, $k_{a}$ is the azimuth signal modulation rate, $T_{d}$ is the target dwell time, $B_{f}, B_{d}$ and $B_{s}$ are bandwidths of the azimuth beam, a point target and the burst, respectively. The 
shadow areas of the TOPS time-frequency diagram (TFD) represent raw data of partly illuminated targets, which are usually considered as invalid data and will be canceled during TOPS SAR focusing.

Figure 5. The impact of the azimuth antenna pattern (AAP) on the AASR and the azimuth loss $L_{a z}$. (a) AASR; (b) Azimuth loss $L_{a z}$.

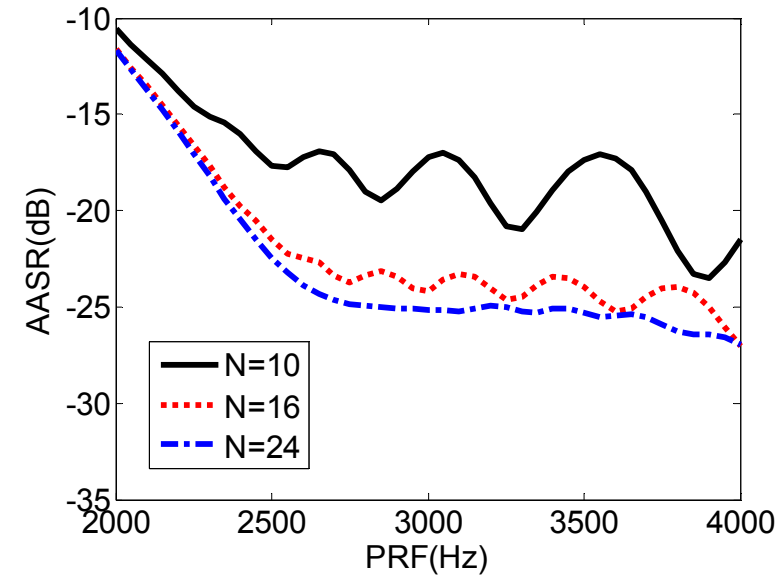

(a)

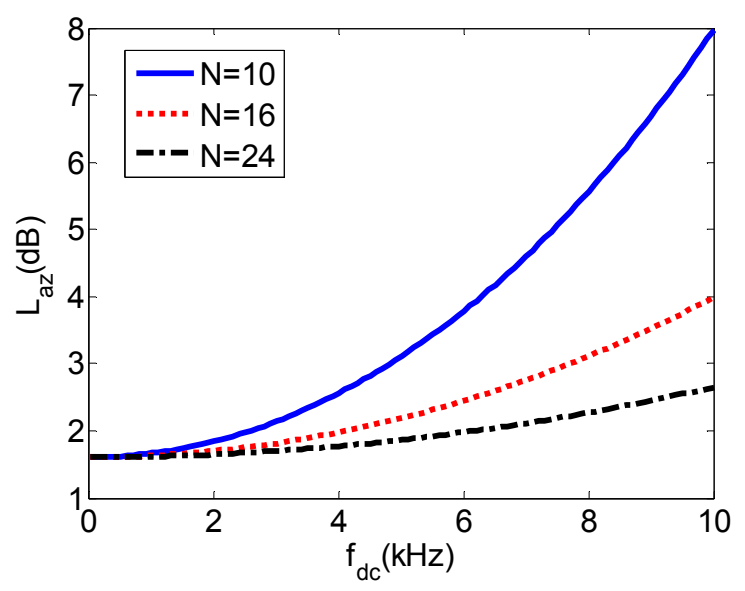

(b)

Figure 6. The TOPS timeline in the azimuth time-frequency diagram.

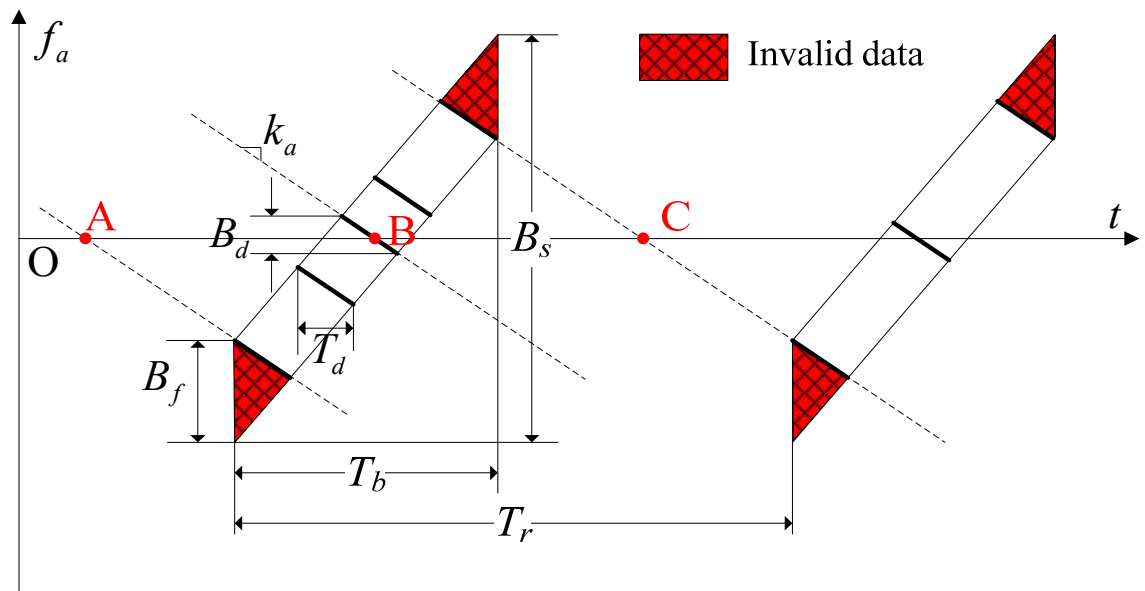

Figure 7. The relationship between cycle time and azimuth resolution.

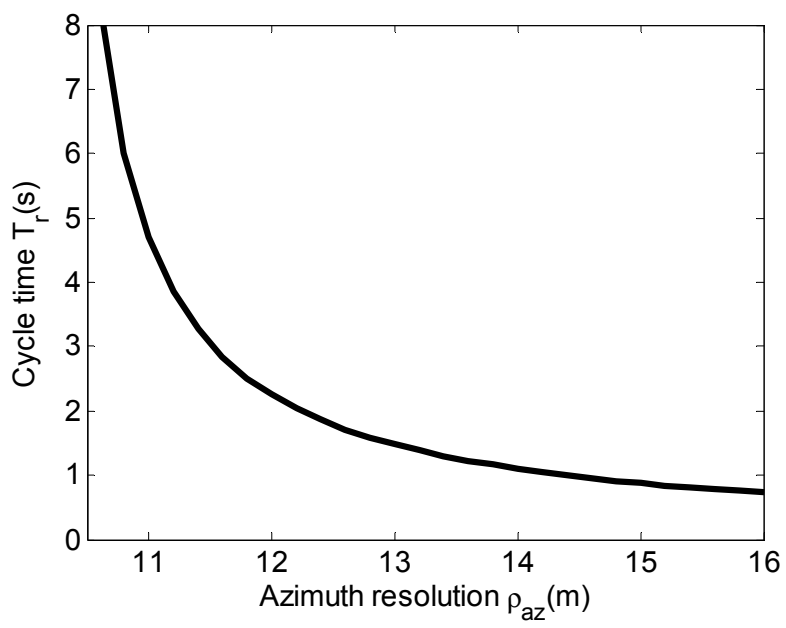


According to Equation (13) and the system parameters listed in Table 1, Figure 7 shows the relationship between the cycle time and the azimuth resolution. It can be seen that a better azimuth resolution needs a larger cycle time, and this indicates that a stronger azimuth beam steering capability is required for a better azimuth resolution in TOPS. However, the azimuth beam steering capability is very limited in recent spaceborne SAR systems, e.g., $\pm 0.75^{\circ}$ in the TerraSAR-X and $\pm 0.7^{\circ}$ in the Sentinel-1 case.

Table 1. Simulation parameters.

\begin{tabular}{cc}
\hline Parameters & Value \\
\hline Height of satellite $H(\mathrm{~km})$ & 630 \\
Satellite velocity $v_{s}(\mathrm{~m} / \mathrm{s})$ & 7,554 \\
Carrier frequency $f_{0}(\mathrm{GHz})$ & 9.65 \\
Look angle $\left({ }^{\circ}\right)$ & $15 \sim 45$ \\
Transmitted pulse width $\tau_{\mathrm{p}}(\mu \mathrm{s})$ & 40 \\
Azimuth antenna length $L_{a}(\mathrm{~m})$ & 6.4 \\
Elevation antenna height $L_{r}(\mathrm{~m})$ & 0.60 \\
Number of Transmit/Receive $(\mathrm{T} / \mathrm{R})$ modules & $16 \times 20$ \\
Transmit peak power $(\mathrm{W})$ & 2,560 \\
Swath width $(\mathrm{km})$ & $\geq 120$ \\
Azimuth resolution $\rho_{a z}(\mathrm{~m})$ & $\leq 15$ \\
Azimuth Ambiguity to Signal Ratio $(\mathrm{AASR})(\mathrm{dB})$ & $\leq-20$ \\
Overlap ratio & $5 \%$ \\
\hline
\end{tabular}

\section{ETOPS Imaging Mode}

To improve the azimuth resolution for spaceborne SAR systems with a limited azimuth beam steering capability, a new burst imaging mode named ETOPS is proposed. The only difference between TOPS and ETOPS modes is that two short extended burst durations are added before and after the conventional TOPS burst duration, respectively. During the extended burst duration, the azimuth beam pointing direction is kept fixed at the center steering angles. The extended burst duration $T_{e}$ is computed as follows:

$$
T_{e}=\frac{B_{d}}{\left|k_{a}\right|}=\frac{\rho_{a}}{\left|k_{a}\right| v_{g}}
$$

where $\rho_{a}$ is the desired azimuth resolution.

Figure 8 demonstrates the ETOPS timeline in the azimuth time-frequency diagram to keep the imaged area continuous in azimuth, and the bottom of Figure 8 shows AAPs seen by point targets with different azimuth positions. The effective azimuth extension Leff_ETOPS of the imaged area for a burst in ETOPS can be expressed as follows:

$$
L_{\text {eff } \_ \text {ETOPS }} \approx\left(\omega_{r} R+v_{g}\right) \cdot\left(T_{b}-2 T_{e}\right)+\vartheta_{0} R=\left(\omega_{r} R+v_{g}\right) \cdot T_{b}-\vartheta_{0} R
$$

Therefore, the constraint of the ETOPS timeline results in a set of $n$ linear equations as follows: 


$$
\left(\omega_{r_{-} i} R_{i}+v_{g}\right) \cdot T_{b_{-} i}-\vartheta_{0} R_{i}=(1+\varepsilon) v_{g} \sum_{i}^{N} T_{b_{-} i}=(1+\varepsilon) v_{g} T_{r}
$$

Compared with Equation (13), the cycle time $T_{r}$ of the ETOPS mode is the same as the one of the TOPS mode. Different from the conventional TOPS mode, the whole burst duration $T_{b}$ includes three parts in the ETOPS mode as shown in Figure 8. The azimuth time interval for azimuth beam steering is just $T_{b}-2 \cdot T_{e}$. Moreover, the azimuth beam is not steered at the burst edges in ETOPS, and the SAR system is working in the squinted stripmap mode. This explains why a smaller azimuth beam scanning capability is required in ETOPS for the same azimuth resolution and range swath width in TOPS.

In Figure 8, point targets A, B and C could not be fully imaged in the conventional TOPS mode, while they would be focused well in ETOPS with the same azimuth beam steering capability. Furthermore, compared to Figure 6, the ratio between the raw data of partly illuminated targets and the whole raw data in ETOPS is obviously lower than the ratio in TOPS. With parameters of sub-swath 1 in TOPS and ETOPS listed in Tables $2-5$, the ratio in TOPS is 0.156 , while the ratio in ETOPS is 0.063 . The bottom of Figure 8 shows the resulting antenna pattern as seen by the point targets at the different azimuth locations.

Figure 8. The Extended Terrain Observation by Progressive Scans (ETOPS) timeline in the azimuth time-frequency diagram.

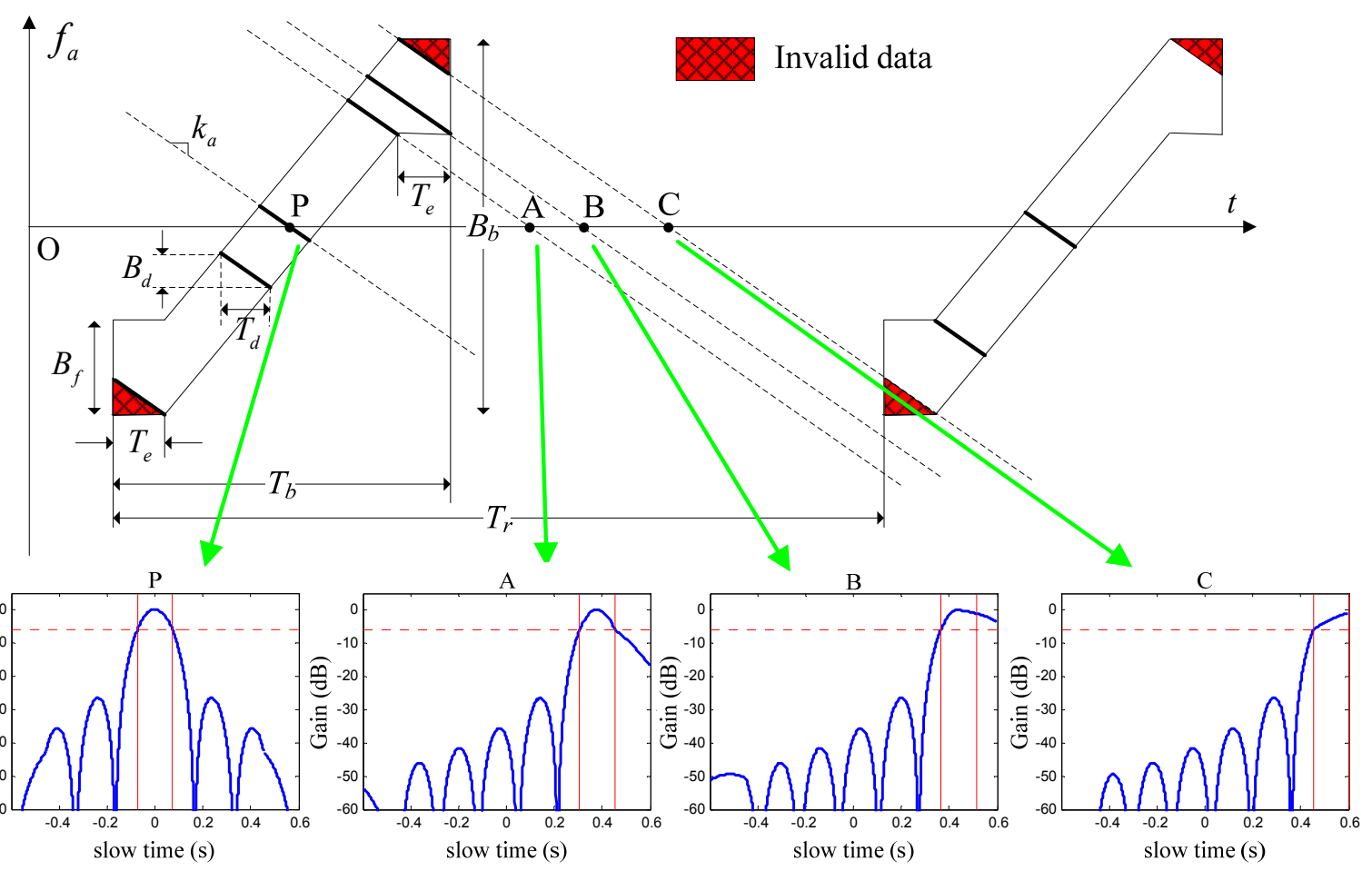

The proposed ETOPS mode in this paper is inspired from another extended TOPS mode named as TOPSPOT [13]. The TOPSPOT is actually a combination of a TOPS SAR and a SPOTSAR scanning, while the proposed ETOPS mode is actually a combination of a TOPS SAR mode and a stripmap mode. The same principle of the TOPSPOT mode and the ETOPS mode is that two short extended burst durations are added before and after the conventional TOPS SAR burst duration. The major difference between the TOPSPOT mode and the ETOPS mode is that the SAR system works in the 
spotlight mode and the azimuth beam requires being steered during the whole extended burst duration in the TOPSPOT mode, while the SAR system works in the stripmap mode and the azimuth beam pointing direction is fixed during the whole extended burst duration in the ETOPS mode. As a result, the AAP illumination of the ETOPS mode for different targets is different from the TOPSPOT mode as shown in Figure 8. Compared with the TOPSPOT mode, targets at the edge of the imaged area in azimuth will be better illuminated.

\section{System Design}

To validate the proposed imaging mode for wide swath coverage, four imaging modes for wide swath coverage with the same system parameters are first designed and their corresponding system performances are analyzed and compared. The major system parameters and system performance requirements are listed in Table 1.

First, a timing analysis is performed. The length of the whole swath consisting of three sub-swaths is $130 \mathrm{~km}$ as shown in Figure 9. With the same timing diagram selection result and system parameters listed in Table 1, four wide width imaging modes (ScanSAR, TOPS, ITOPS and ETOPS) are designed. The parameters of the different sub-swaths for the different modes are computed and summarized in Tables 2-5.

Figure 9. The timing diagram for wide-swath imaging with three-sub-swaths according to Table 1.

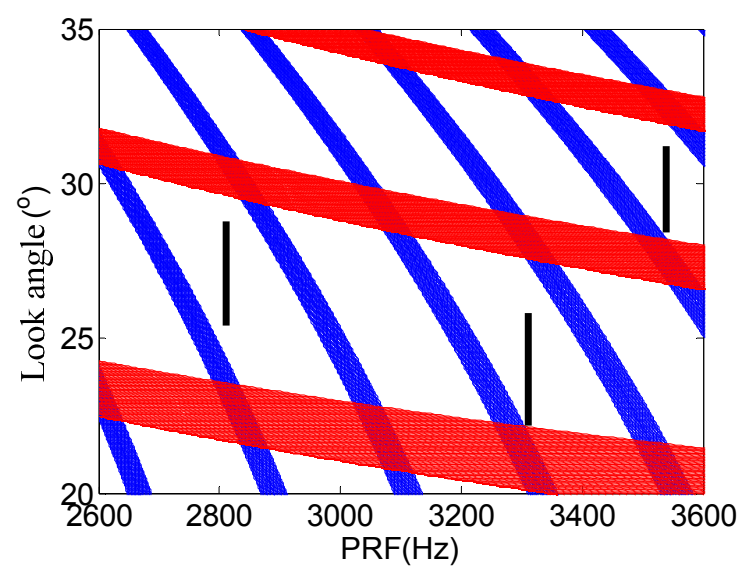

Table 2. Parameters of different sub-swaths in ScanSAR.

\begin{tabular}{cccc}
\hline Sub-Swath & Sub-Swath-1 & Sub-Swath-2 & Sub-Swath-3 \\
\hline Burst duration $(\mathrm{s})$ & 0.103 & 0.106 & 0.109 \\
Echoes per burst & 340 & 297 & 385 \\
Target dwell time $T_{d}(\mathrm{~s})$ & 0.103 & 0.106 & 0.109 \\
Target bandwidth $B_{d}(\mathrm{~Hz})$ & 489.78 & 489.62 & 489.58 \\
Burst bandwidth $B_{b}(\mathrm{kHz})$ & 2.088 & 2.088 & 2.088 \\
Azimuth extension $L_{\text {eff }}(\mathrm{km})$ & 2.296 & 2.365 & 2.434 \\
Azimuth resolution $\rho_{a}(\mathrm{~m})$ & 14 & 14 & 14 \\
\hline
\end{tabular}


Table 3. Parameters of different sub-swaths in Terrain Observation by Progressive Scans (TOPS).

\begin{tabular}{cccc}
\hline Sub-Swath & Sub-Swath-1 & Sub-Swath-2 & Sub-Swath-3 \\
\hline Burst duration $T_{b}(\mathrm{~s})$ & 0.748 & 0.751 & 0.755 \\
Echoes per burst & 2474 & 2110 & 2671 \\
Target dwell time $T_{d}(\mathrm{~s})$ & 0.117 & 0.120 & 0.124 \\
Beam steering rate $\omega_{r}\left({ }^{\circ} / \mathrm{s}\right)$ & 1.550 & 1.505 & 1.462 \\
Max steering angle $\theta_{\max }\left({ }^{\circ}\right)$ & 0.579 & 0.565 & 0.552 \\
Target bandwidth $B_{d}(\mathrm{~Hz})$ & 571.4 & 571.2 & 571.2 \\
Burst bandwidth $B_{b}(\mathrm{kHz})$ & 11.009 & 10.787 & 10.579 \\
Azimuth extension $L_{e f f}(\mathrm{~km})$ & 16.222 & 16.217 & 16.216 \\
Azimuth resolution $\rho_{a}(\mathrm{~m})$ & 12 & 12 & 12 \\
\hline
\end{tabular}

Table 4. Parameters of different sub-swaths in inverse TOPS (ITOPS).

\begin{tabular}{cccc}
\hline Sub-Swath & Sub-Swath-1 & Sub-Swath-2 & Sub-Swath-3 \\
\hline Burst duration $T_{b}(\mathrm{~s})$ & 0.748 & 0.751 & 0.755 \\
Echoes per burst & 2474 & 2110 & 2671 \\
Target dwell time $T_{d}(\mathrm{~s})$ & 0.117 & 0.120 & 0.124 \\
Beam steering rate $\omega_{r}\left({ }^{\circ} / \mathrm{s}\right)$ & 2.677 & 2.599 & 2.526 \\
Max steering angle $\theta_{\max }\left({ }^{\circ}\right)$ & 1.001 & 0.976 & 0.953 \\
Target bandwidth $B_{d}(\mathrm{~Hz})$ & 571.4 & 571.2 & 571.2 \\
Burst bandwidth $B_{b}(\mathrm{kHz})$ & 17.496 & 17.113 & 16.754 \\
Azimuth extension $L_{\text {eff }}(\mathrm{km})$ & 16.222 & 16.217 & 16.216 \\
Azimuth resolution $\rho_{a}(\mathrm{~m})$ & 12 & 12 & 12 \\
\hline
\end{tabular}

Table 5. Parameters of different sub-swaths in Extended Terrain Observation by Progressive Scans (ETOPS).

\begin{tabular}{|c|c|c|c|}
\hline $\begin{array}{ll}\text { Parameters } & \text { Sub-Swath } \\
\end{array}$ & Sub-Swath-1 & Sub-Swath-2 & Sub-Swath-3 \\
\hline Burst duration $T_{b}(\mathrm{~s})$ & 0.748 & 0.751 & 0.755 \\
\hline Echoes per burst & 2474 & 2110 & 2671 \\
\hline Extended burst duration $T_{e}(\mathrm{~s})$ & 0.117 & 0.120 & 0.124 \\
\hline Target dwell time $T_{d}(\mathrm{~s})$ & 0.117 & 0.120 & 0.124 \\
\hline Beam steering rate $\omega_{r}(\%)$ & 1.550 & 1.505 & 1.462 \\
\hline Max steering angle $\theta_{\max }\left({ }^{\circ}\right)$ & 0.398 & 0.385 & 0.371 \\
\hline Target bandwidth $B_{d}(\mathrm{~Hz})$ & 571.4 & 571.2 & 571.2 \\
\hline Burst bandwidth $B_{b}(\mathrm{kHz})$ & 9.123 & 8.888 & 8.652 \\
\hline Azimuth extension $L_{e f f}(\mathrm{~km})$ & 16.222 & 16.217 & 16.216 \\
\hline Azimuth resolution $\rho_{a}(\mathrm{~m})$ & 12 & 12 & 12 \\
\hline
\end{tabular}

With the same system parameters, the obtained azimuth resolution is $14 \mathrm{~m}$ in ScanSAR, while the obtained azimuth resolution of the other modes is $12 \mathrm{~m}$. This phenomenon is caused by different imaging principles to keep the imaged area continuous in azimuth. For the same desired azimuth resolution, the maximum azimuth steering angle in TOPS, ITOPS and ETOPS are $0.579^{\circ}, 1.001^{\circ}$ and $0.398^{\circ}$, respectively, and the ETOPS mode requires a lower azimuth beam steering angle than the other two modes. 
Figure 10 shows the azimuth ambiguity to signal ratio (AASR) of the different imaging modes a function of the target along-track position. The azimuth variant AASR is the most obvious in ScanSAR, since echoes of different targets are weighted by different parts of the AAP depending on the target's azimuth locations. The slight AASR variation along the azimuth direction in TOPS and ITOPS is caused by the increased grating lobes and the main lobe reduction during azimuth beam steering. This effect is more obvious in ITOPS than in TOPS, since a higher steering angle is required for a desired azimuth resolution. As the azimuth beam pointing direction is fixed during the extended burst duration in ETOPS, the azimuth variant AASR is obvious at the edge of the imaged area in azimuth, but it is less obvious than ScanSAR as shown in Figure 10d.

Figure 10. AASR of different imaging modes. (a) ScanSAR; (b) TOPS; (c) ITOPS; (d) ETOPS.

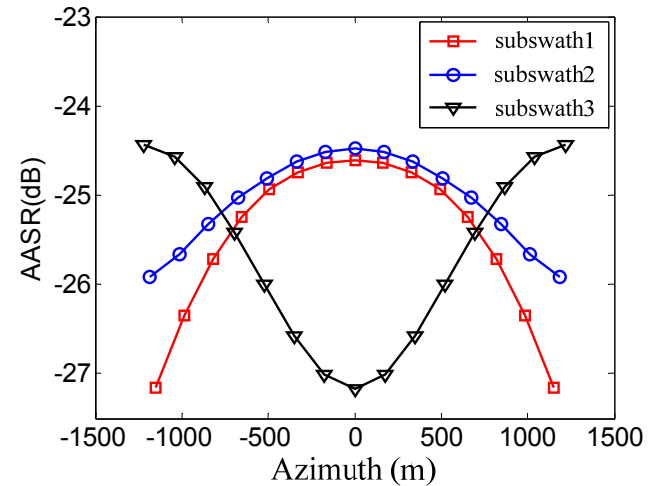

(a)

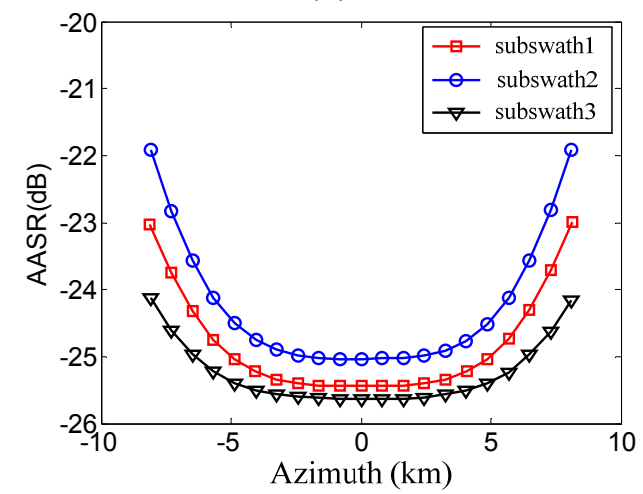

(c)

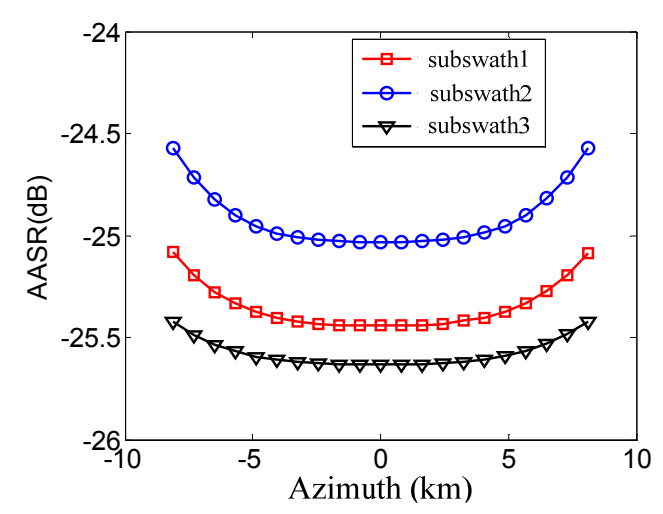

(b)

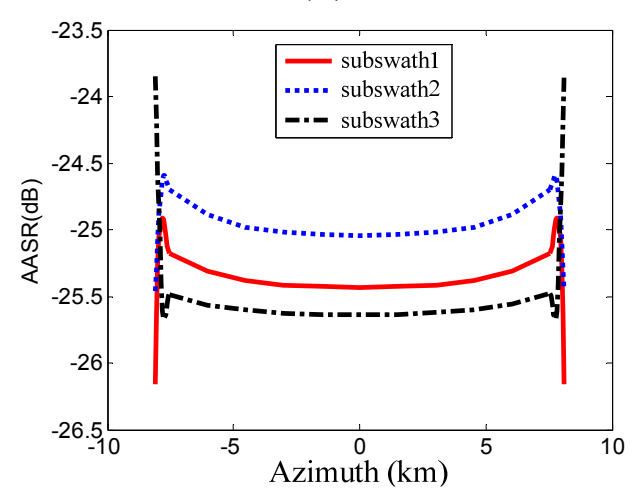

(d)

Noise-equivalent sigma zero (NESZ) is also a function of the target along-track position. In ScanSAR, this variation exceeds $3 \mathrm{~dB}$ as shown in Figure 11a, since echoes of targets with different azimuth locations are weighted by different parts of the AAP. This variation is less than $0.3 \mathrm{~dB}$ in TOPS and less than $1.2 \mathrm{~dB}$ in ITOPS, since it is caused by the antenna gain reduction during azimuth beam steering. In ETOPS, the NESZ variation in azimuth is about $2 \mathrm{~dB}$ and better than that of ScanSAR.

Performance analysis results as shown in Figures 10 and 11 validate the proposed ETOPS mode. The azimuth performance variation in ETOPS is better than in ScanSAR. Furthermore, for the same azimuth resolution, the ETOPS mode requires a smaller azimuth beam steering angle than the TOPS and ITOPS modes, which also means that a better azimuth resolution could be obtained in ETOPS for a limited azimuth beam steering capability. 
Figure 11. Noise-equivalent sigma zero (NESZ) of different imaging modes. (a) ScanSAR; (b) TOPS; (c) ITOPS; (d) ETOPS.

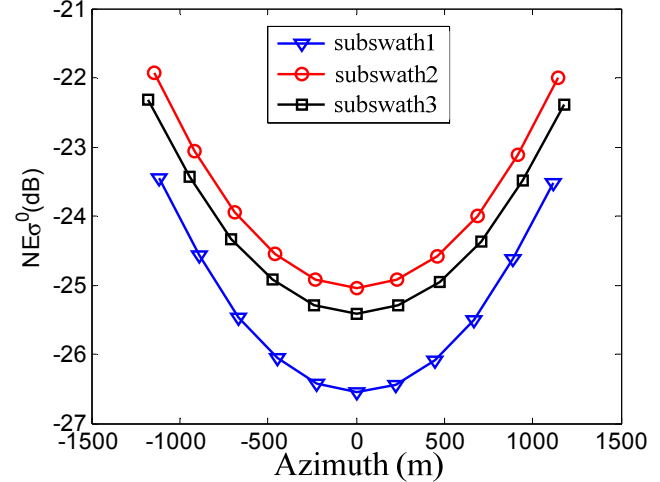

(a)

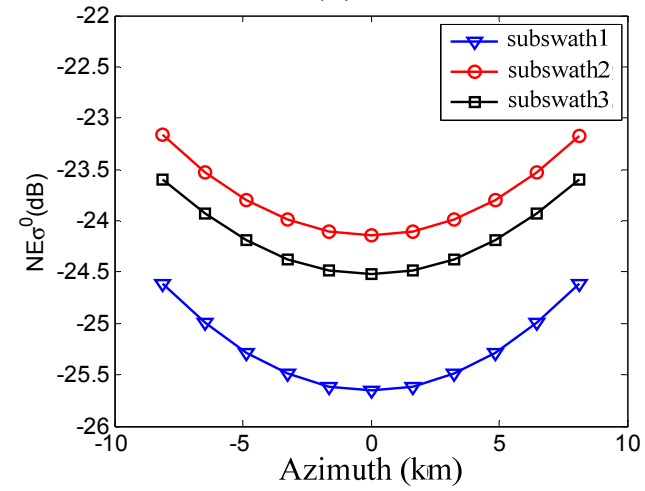

(c)

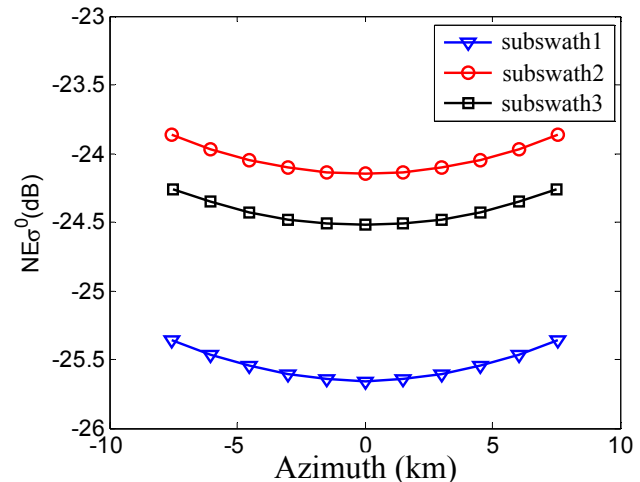

(b)

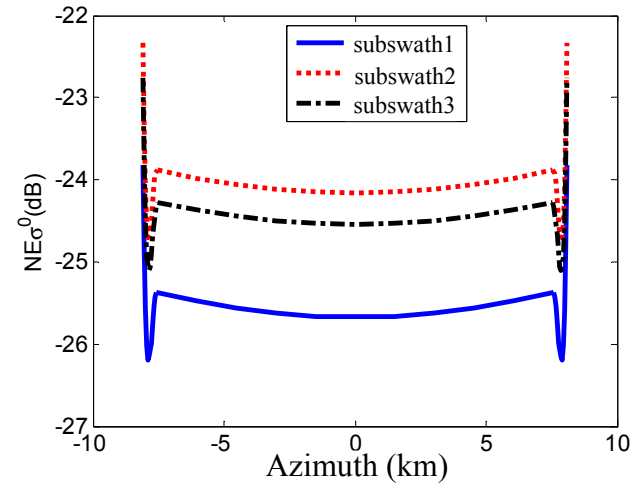

(d)

\section{Conclusions}

Burst imaging schemes are usually adopted for wide swath coverage. This paper proposes a new burst imaging scheme named ETOPS. Performance (AASR and NESZ) of the ETOPS are better than ScanSAR, but little worse than the TOPS mode. However, with a limited azimuth beam scanning capability, the ETOPS mode could implement a better azimuth resolution than the TOPS mode. Furthermore, the ratio between the raw data of partly illuminated targets and the whole raw data in ETOPS is obviously lower than the ratio in TOPS. Four different burst imaging modes for wide swath coverage have been designed with the same system parameters, and their corresponding performances are analyzed and compared. Simulation results validate the proposed ETOPS mode for wide swath coverage.

\section{Acknowledgments}

This work was supported by the National Natural Science Foundation of China under Grant 61201433.

\section{Author Contributions}

This paper was written by Pingping Huang, and Wei Xu gave some suggestions for performance simulation, especially for the TOPS mode. 


\section{Conflicts of Interest}

The authors declare no conflict of interest.

\section{References}

1. Currie, A.; Brown, M.A. Wide-swath SA. Proc. Inst. Elect. Eng. F 1992, 139, 123-135.

2. Moore, R.K.; Claassen, J.P.; Lin, Y.H. Scanning spaceborne syn-thetic aperture radar with integrated radiometer. IEEE Trans. Aerosp. Electron. Syst. 1981, 17, 410-420.

3. Guarnieri, A.M.; Prati, C. ScanSAR focussing and interferome-try. IEEE Trans. Geosci. Remote Sens. 1996, 34, 1029-1038.

4. Holzner, J.; Bamler, R. Burst-Mode and ScanSAR Interferometry. IEEE Trans. Geosci. Remote Sens. 2002, 40, 1917-1934.

5. Rosen, P.; Hensley, S.; Joughin, I.R.; Li, F.K.; Madsen, S.; Rodríguez, E.; Goldstein, R. Synthetic aperture radar interferometry. Proc. IEEE 2000, 88, 333-382.

6. Zan, F.D.; Guarnieri, A.M. TOPSAR: Terrain observation by progressive scans. IEEE Trans. Geosci. Remote Sens. 2006, 44, 2352-2360.

7. Xu, W.; Huang, P.-P.; Deng, Y.-K. TOPSAR data focusing based on azimuth scaling preprocessing. Adv. Space Res. 2011, 48, 270-277.

8. Xu, W.; Huang, P.-P.; Deng, Y.-K.; Sun, J.-T.; Shang, X.-Q. An efficient imaging approach with scaling factors for TOPS mode SAR data focusing. IEEE Geosci. Remote Sens. Lett. 2011, 8, 929-933.

9. Meta, A.; Mittermayer, J.; Prats, P.; Scheiber, R.; Steinbrecher, U. TOPS imaging with TerraSAR-X: Mode design and perfomance analysis. IEEE Trans. Geosci. Remote Sens. 2010, 48, 759-769.

10. Meta, A.; Prats, P.; Steinbrecher, U.; Mittermayer, J.; Scheiber, R. TerraSAR-X TOPS and ScanSAR Comparison. In Proceedings of the 7th European Conference on Synthetic Aperture Radar (EUSAR), Friedrichshafen, Germany, 2-5 June 2008.

11. Attema, E.; Davidson, M.; Floury, N.; Levrini, G.; Rosich, B.; Rommen, B.; Snoeij, P. Sentinel-1 ESA's New European Radar Observatory. In Proceedings of the 7th European Conference on Synthetic Aperture Radar (EUSAR), Friedrichshafen, Germany, 2-5 June 2008; Volume 2, pp. 179-182.

12. $\mathrm{Xu}, \mathrm{W}$.; Deng, Y.K. Investigation on electronic azimuth beam steering in the spaceborne SAR imaging modes. J. Electromagn. Wave. Appl. 2011, 25, 2076-2088.

13. D'Aria, D.; de Zan, F.; Giudici, D.; Guarnieri, A.M.; Rocca, F. Burst-mode SARs for wide-swath surveys. Can. J. Remote Sens. 2007, 33, 27-38.

14. Wollstadt, S.; Prats, P.; Bachmann, M.; Mittermayer, J.; Scheiber, R. Scalloping correction in TOPS imaging mode SAR data. IEEE Geosci. Remote Sens. Lett. 2012, 9, 614-618.

(C) 2014 by the authors; licensee MDPI, Basel, Switzerland. This article is an open access article distributed under the terms and conditions of the Creative Commons Attribution license (http://creativecommons.org/licenses/by/3.0/). 